\title{
Alteration of plasma metabolites associated with chemoradiosensitivity in esophageal squamous cell carcinoma via untargeted metabolomics approach
}

Yaowen Zhang ${ }^{1 \dagger}$, Jianpo Wang ${ }^{1 \dagger}$, Ningtao Dai ${ }^{1}$, Peng Han ${ }^{1}$, Jian $\mathrm{Li}^{1}$, Jiangman Zhao ${ }^{2}$, Weilan Yuan ${ }^{2}$, Jiahuan Zhou $^{2^{*}}$ and Fuyou Zhou ${ }^{1 *}$

\begin{abstract}
Background: To investigate the differences in plasma metabolomic characteristics between pathological complete response ( $p C R$ ) and non-pCR patients and identify biomarker candidates for predicting the response to neoadjuvant chemoradiotherapy (nCRT) in esophageal squamous cell carcinoma (ESCC).

Methods: A total of 46 ESCC patients were included in this study. Gas chromatography time-of- flight mass spectrometry (GC-TOF/MS) technology was applied to detect the plasma samples collected before nCRT via untargeted metabolomics analysis.

Results: Five differentially expressed metabolites (out of 109) was found in plasma between $p C R$ and non-pCR groups. Compared with non-pCR group, isocitric acid $(p=0.0129)$, linoleic acid $(p=0.0137)$, citric acid $(p=0.0473)$ were upregulated, while L-histidine $(p=0.0155), 3^{\prime} 4$ dihydroxyhydrocinnamic acid $(p=0.0339)$ were downregulated in the pCR plasma samples. Pathway analyses unveiled that citrate cycle (TCA cycle), glyoxylate and dicarboxylate metabolic pathway were associated with ESCC chemoradiosensitivity.
\end{abstract}

Conclusion: The present study provided supporting evidence that GC-TOF/MS based metabolomics approach allowed identification of metabolite differences between $\mathrm{pCR}$ and non-pCR patients in plasma levels, and the systemic metabolic status of patients may reflect the response of ESCC patient to neoadjuvant chemoradiotherapy.

Keywords: Chemoradiosensitivity, Esophageal squamous cell carcinoma, Metabolomics, Neoadjuvant therapy, Untargeted metabolomics analysis

\footnotetext{
*Correspondence: zhoujh@biotecan.com; ayzhoufuyou@163.com

†Yaowen Zhang and Jianpo Wang contributed equally to this work.

${ }^{2}$ Shanghai Zhangjiang Institue of Medical Innovation, Shanghai Biotecan Pharmaceuticals Co., Ltd., 180 Zhangheng Road, Shanghai 201204, China

${ }^{1}$ Anyang Cancer Hospital, The 4th Affiliated Hospital of Henan University of Science and Technology, No.1 Huanbin North Road, Anyang 455000, Henan Province, China
}

C C The Author(s). 2020 Open Access This article is licensed under a Creative Commons Attribution 4.0 International License, which permits use, sharing, adaptation, distribution and reproduction in any medium or format, as long as you give appropriate credit to the original author(s) and the source, provide a link to the Creative Commons licence, and indicate if changes were made. The images or other third party material in this article are included in the article's Creative Commons licence, unless indicated otherwise in a credit line to the material. If material is not included in the article's Creative Commons licence and your intended use is not permitted by statutory regulation or exceeds the permitted use, you will need to obtain permission directly from the copyright holder. To view a copy of this licence, visit http://creativecommons.org/licenses/by/4.0/. The Creative Commons Public Domain Dedication waiver (http://creativecommons.org/publicdomain/zero/1.0/) applies to the data made available in this article, unless otherwise stated in a credit line to the data. 


\section{Background}

Esophageal cancer (EC) as an aggressive malignant tumor, is the sixth leading cause of cancer death globally [1]. Over $50 \%$ of all EC-related deaths occur in China where esophageal squamous cell carcinoma (ESCC) is the predominant histologic subtype [2]. Surgery is the primary treatment of esophageal cancer, especially for patients with early stage [3, 4]. But most esophageal cancer cases has progressed to advanced stage before they are finally diagnosed [5]. Neoadjuvant chemoradiotherapy (nCRT) has been considered as a promising therapy strategy for patients with stage II or III esophageal cancer. Several studies have shown that neoadjuvant chemoradiotherapy plus surgery contributes to improved local control, progression free survival, and overall survival compared with surgery alone [6-8]. However, not all the EC patients could benefit from nCRT and poor responders have to experience severe toxicity and impaired quality of life $[9,10]$. Moreover, the outcomes of nonresponders were found to be worse than those underwent primary resection [11]. Hence, it is essential to find a reliable marker of chemoradiosensitivity in esophageal cancer to avoid wasting valuable time and obtain a more favorable prognosis for patients.

Metabolomics have been widely applied in diagnosis and biomarker screening study on various disease, including cancer $[12,13]$. Since metabolites represent the end products of biochemical processes, it is closely linked to the overall physiopathological status of an individual [14]. It has been discovered that alterations of metabolites in the biofluids (serum, plasma, and urine) are related to prognosis [15], recurrence [16], treatment response [17] in cancer patients. So far, metabolomic studies on EC have been performed to identify differential metabolite markers between patients and controls [18]. In addition, multiple metabolites have been found to be strongly associated with the degree of tumor progression through metabolomics-based methods [19, 20]. As for the biomarker screening for chemoradiosensitivity in ESCC, one research with small sample size revealed that serum levels of several metabolites differed significantly between the pathological complete response (pCR) group and non-pCR group [21]. However, no other external validation was provided.

In the present study, we aim to investigate the differences in plasma metabolomic characteristics between pCR and non-pCR patients and identify biomarker candidates for predicting the response to neoadjuvant chemoradiotherapy (nCRT) in esophageal squamous cell carcinoma (ESCC). We used gas chromatography timeof-flight mass spectrometry (GC-TOF/MS) technology which is more conducive to the rapid detection of complex samples analysis for untargeted metabolic profiling. The results showed that five metabolites demonstrated differences between $\mathrm{pCR}$ and non-pCR patients in the plasma collected before the onset of neoadjuvant therapy. And pathway analyses unveiled that citrate cycle, glyoxylate and dicarboxylate metabolic pathway were associated between pCR and non-pCR groups. This study provided supporting evidence that GC-TOF/MS based metabolomics approach allowed identification of metabolite differences between $\mathrm{pCR}$ and non-pCR patients in plasma levels, and the systemic metabolic status of patients may reflect the response of ESCC to neoadjuvant chemoradiotherapy.

\section{Methods}

\section{Sample collection}

The study included plasma samples from 46 stage II-III esophageal cancer patients who were prospectively selected at the Anyang Cancer Hospital (Henan, China) between June 2017 and April 2019. All patients had been pathologically diagnosed esophageal squamous cell carcinoma. The neoadjuvant chemoradiotherapy (nCRT) consisted of radiotherapy (total radiation dose: 45Gy, 1.8Gy/day, 25 fractions) and concurrent chemotherapy with paclitaxel $\left(135-150 \mathrm{mg} / \mathrm{m}^{2}\right)$ plus cisplatin $(50-75$ $\mathrm{mg} / \mathrm{m}^{2}$ ) every 21 days for two cycles. 4-6 weeks after completion of nCRT, patients underwent surgery. Clinical stages and pathological stages were determined according to the eighth edition of the American Joint Committee on Cancer tumor-node-metastasis (TNM) staging criteria [22]. All pathology slides were reviewed by a pathologist to determine the pathologic response. pCR was defined as no evidence of viable tumor cells in all specimens, including the primary site and lymph nodes [23]. Samples were collected just before the onset of neoadjuvant therapy and kept frozen and stored at $80{ }^{\circ} \mathrm{C}$ for further analysis. This study was approved by the Ethics Committee of Anyang Cancer Hospital.

\section{Sample preparation for metabolomic analysis}

The untargeted metabolomics profiling was implemented on XploreMET platform (Metabo-Profile, Shanghai, China). The sample preparation was conducted as their published methods with minor modifications [24, 25]. In brief, the plasma samples were centrifuged at $3000 \times g$ and $4{ }^{\circ} \mathrm{C}$ for $5 \mathrm{~min}$ (Microfuge 20R, Beckman Coulter, Inc., Indianapolis, IN, USA) after thawing to separate debris or a lipid layer. Metabolites were extracted from plasma samples $(50 \mu \mathrm{L})$ with $10 \mu \mathrm{L}$ of internal standard (0.5 mM 4-Chlorophenylalanine) and $175 \mu \mathrm{L}$ of pre-chilled methanol: chloroform (3:1) followed by centrifugation at $14,000 \times \mathrm{g}$ for $20 \mathrm{~min}$ at $4{ }^{\circ} \mathrm{C}$. Then each $200 \mu \mathrm{L}$ of the supernatant was transferred into an autosampler vial (Agilent Technologies, Foster City, CA, USA). The resting supernatant from each sample was pooled to prepare quality control 
samples. Following solvent evaporation and lyophilization, the dried samples were derivatized with $50 \mu \mathrm{L}$ of methoxyamine ( $20 \mathrm{mg} / \mathrm{ml}$ in pyridine) for $2 \mathrm{~h}$, followed by silylanization with $50 \mu \mathrm{L}$ of MSTFA (1\% TMCS) for $1 \mathrm{~h}$ prior to injection. Above two steps were performed by a robotic multipurpose sample MPS2 with dual heads (Gerstel, Muehlheim, Germany).

\section{Metabolomic analysis}

The GC-TOF/MS analysis was performed using a timeof-flight mass spectrometry (GC-TOF/MS) system (Pegasus HT, Leco Corp., St. Joseph, MO,USA), which consists of an Agilent 7890B gas chromatography and a Gerstel multipurpose sample MPS2 with dual heads (Gerstel, Muehlheim, Germany). As described previously [26], DB-5MS GC column $(30 \mathrm{~m} \times 250 \mu \mathrm{m}$ i.d., $0.25-\mu \mathrm{m}$ film thickness; Restek corporation, Bellefonte, PA, USA) was chosen for separation. Helium was used as the carrier gas at a steady flow rate of $1.0 \mathrm{~mL} / \mathrm{min}$. The temperature of transfer interface and injection were both $270^{\circ} \mathrm{C}$. The source temperature was set as $220^{\circ} \mathrm{C}$. The measurements were taken using electron impact ionization $(70 \mathrm{eV})$ in the full scan mode $(\mathrm{m} / \mathrm{z} 50-500)$.

\section{Data processing}

XploreMET (v3.0, Metabo-Profile, Shanghai, China) was used to process the raw data generated by GC-TOF/MS. The data processing includes baseline denosing and smoothing, peak picking and deconvultion, creating reference database from the pooled QC samples, metabolite signal alignment, missing value correction and imputation, and QC correction as previously reported [27]. Metabolites were identified by comparing both retention index and mass spectral data with JiaLibTM metabolite database. Each data set was converted into comparable data vectors for statistical analysis. The metabolites with $\mathrm{t}$ test or $\mathrm{U}$ test $\mathrm{P}<0.05$ by unidimensional analysis were considered as differentially expressed between pCR and non-pCR groups.

\section{Pathway analyses}

To further investigate the metabolic pathways involved in the chemoradiosensitivity in ESCC, the differential metabolites were annotated with Kyoto Encyclopedia of Genes and Genomes (KEGG, http://www.genome.jp/ $\mathrm{kegg} /$ ) and Human Metabolome Database (HMDB, http://www.hmdb.ca/). MetaboAnalyst 4.0 (http://www. metaboanalyst.ca/MetaboAnalyst/) were then applied to analyze the data by $\mathrm{R}$ software (v3.4.3, GitHub). The iPath 3.0 (http://pathways.embl.de/) was used to show the metabolic network of the differential metabolites and altered metabolic pathways in KEGG general metabolic pathway.

\section{Statistical analysis}

All measurements were mean-centered and scaled by the standard deviation of the observed measurements. Fisher's exact probability test, the Student's t-test, or the Mann-Whitney U-test was used to evaluate the statistical significance of differences. The Mann-Whitney $U$ test was utilized for non-normal distribution data and the Student's t-test was applied for normal distribution data. Fisher's exact tests for categorical variables. The area under the receiver operating characteristic (ROC) curve (AUC) was performed to assess the feasibility of using the plasma levels of particular metabolites as predictive biomarkers. The data was randomly split into a training set (data from 36 patients) and a test set (data from 10 patients). Predictions were made on the test set based on the Gaussian Naive Bayes model trained in the training set. $P<0.05$ was considered statistically significant.

\section{Results}

Patients \& treatment outcomes

A total of 46 patients were included in this study. The age of all subjects ranged from 50 to 84 years, including 30 men and 16 women. According to the American Joint Committee on Cancer tumor-node-metastasis (TNM)

Table 1 Clinical characteristics of the patients

\begin{tabular}{|c|c|c|c|c|}
\hline \multirow{2}{*}{$\begin{array}{l}\text { Clinical } \\
\text { characteristics }\end{array}$} & \multirow{2}{*}{$\begin{array}{l}\text { Case } \\
n=46\end{array}$} & \multicolumn{2}{|c|}{ Histological response } & \multirow[t]{2}{*}{$P$ values } \\
\hline & & $\begin{array}{l}\text { non-pCR } \\
(n=23)\end{array}$ & $\begin{array}{l}\text { pCR } \\
(n=23)\end{array}$ & \\
\hline Gender & & & & 0.758 \\
\hline Male & 30 & 16 & 14 & \\
\hline Female & 16 & 7 & 9 & \\
\hline Age & & & & 1.000 \\
\hline$<65$ & 19 & 9 & 10 & \\
\hline$\geq 65$ & 27 & 14 & 13 & \\
\hline Tumor location & & & & $0.041^{*}$ \\
\hline Cervical & 2 & 2 & 0 & \\
\hline $\mathrm{Lt}$ & 7 & 5 & 2 & \\
\hline Mt & 23 & 13 & 10 & \\
\hline Ut & 14 & 3 & 11 & \\
\hline TNM stage & & & & $0.028^{*}$ \\
\hline$\|$ & 4 & 0 & 4 & \\
\hline III & 42 & 23 & 19 & \\
\hline Smoking & & & & 0.136 \\
\hline Yes & 20 & 13 & 7 & \\
\hline No & 26 & 10 & 16 & \\
\hline Drinking & & & & 1.000 \\
\hline Yes & 16 & 8 & 8 & \\
\hline No & 30 & 15 & 15 & \\
\hline
\end{tabular}


staging criteria (the eighth edition), the pre-treatment clinical stages of these 46 subjects were II $(n=4)$ and III $(n=42)$, respectively. After nCRT, 23 patients achieved pCR through the examination of surgical pathology specimen, while the remaining 23 patients were identified into non-pCR group. The baseline characteristics of the ESCC patients and treatment outcomes are shown in Table 1. According to Fisher's exact probability test, tumor location and clinical stage are significantly different between the $\mathrm{pCR}$ and non-pCR groups. There were no significant differences in gender, age, smoking and alcohol consumption between the two groups.

\section{Altered metabolites in plasma among $\mathrm{pCR}$ and non- $\mathrm{pCR}$ patients}

A total of 109 metabolites of different classes (Amino Acids, Organic Acids, Carbohydrates, Phosphates, Fatty Acids, Indoles) were detected in the plasma samples from 46 subjects using untargeted metabolomics analysis (Fig. 1a and Supplementary List 1). To further determine

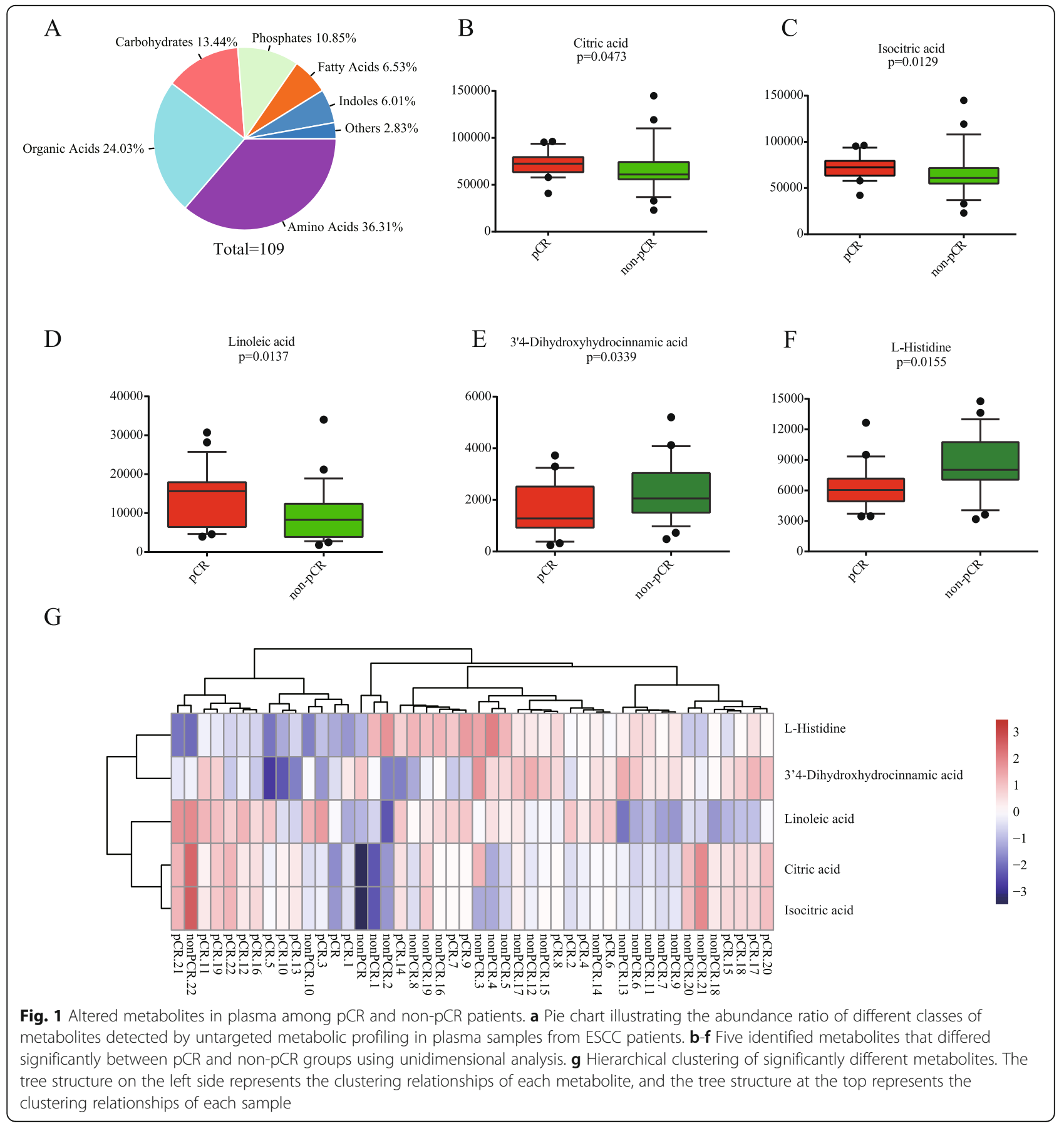


whether the metabolite footprints in plasma differed between $\mathrm{pCR}$ and non-pCR subjects, unidimensional analysis was used (Student's t-test or Mann-Whitney U test was selected according to the data) to obtain the differential metabolites between the two groups. We found five differentially expressed metabolites (out of 109) in plasma levels between pCR and non-pCR groups. Compared with non-pCR group, isocitric acid, linoleic acid, citric acid were upregulated $(p=0.0129,0.0137$ and 0.0473, respectively), and L-histidine, 3'4-Dihydroxyhydrocinnamic acid were downregulated $(p=0.0 .0155$ and 0.0339 , respectively) in the pCR plasma samples (Fig. 1b-f). In order to display the relationships among samples and differences in expression patterns more intuitively, we also conducted the bidirectional clustering analysis of each samples and the significantly different metabolites. As the results showed that the samples in two groups can be distinguished, and metabolites between two groups existed difference (Fig. 1g).

Furthermore, ROC analysis was applied to explore whether the five differential metabolites could be converted into an approach for predicting nCRT response. As the ROC curve performed by Gaussian Naive Bayes model showed that the area under the curve (AUC) value was 0.76 (Fig. 2), which implied a good ability in predicting the response of $\mathrm{pCR}$ and non-pCR of esophageal cancer patients by these five metabolites.

\section{Metabolic pathway analysis}

To further explore the metabolic pathways related to above ESCC chemoradiosensitivity-associated metabolites, MetaboAnalyst was used to indicate the metabolic pathways connected with these five metabolites. Through pathway analysis based on KEGG database, two pathways namely citrate cycle (TCA cycle), glyoxylate and dicarboxylate metabolism were altered in pCR patients (Fig. 3 and Table 2). The overall metabolic network of the altered metabolites and metabolic pathways is shown in Supplementary Figure 1.

\section{Discussion}

Although the benefit of nCRT has been demonstrated in multiple trials, only approximately $20-25 \%$ of EC patients showed complete response, and some even developed resistance to currently used therapy [28]. Chemoradiotherapy resistance is a considerable obstacle to the effective treatment of esophageal cancer. Unfortunately, we have not yet identified specific biomarkers in blood which would reliably and accurately predict response to nCRT.

Metabolism alterations in cancer are well-documented. Metabolomics as a new high throughput technology provides a broad field for finding metabolites as potential biomarkers in body fluids for diagnosis, treatment and prognosis of cancer. Gas chromatography time-of-flight mass spectrometry (GC-TOF-MS) with its pivotal characteristics, including higher selectivity, resolution, sensitivity and accuracy is well-suited for the identification and quantitation of low molecular weight metabolites $[29,30]$. In the present study, GC-TOF/MS-based untargeted metabonomics approach was utilized to profile metabolites in ESCC plasma samples from the pCR and non-pCR patients.

The major findings of this study were summarized as follows: i) A total of five key metabolites associated with ESCC chemoradiosensitivity were identified; ii) Among the five differential metabolites, isocitric acid, linoleic acid and citric acid were upregulated, while L-histidine, 3'4 dihydroxyhydrocinnamic acid were downregulated (Fig. 1b-f); iii) Multiple metabolic pathways, especially
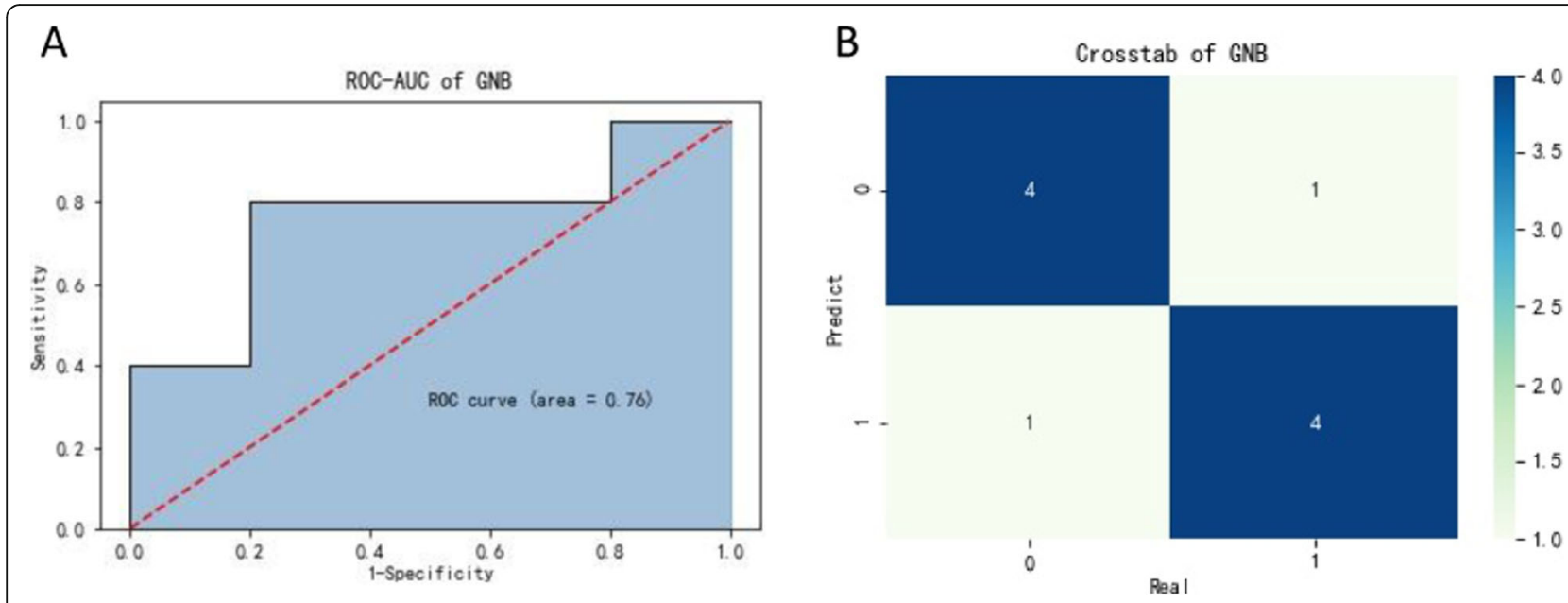

Fig. 2 Evaluation of the five differential metabolites in predictive precision. a ROC curve based on the five differentially expressed plasma metabolites. b Confusion matrix showing classification results ( $80 \%$ overall correct assignment) 


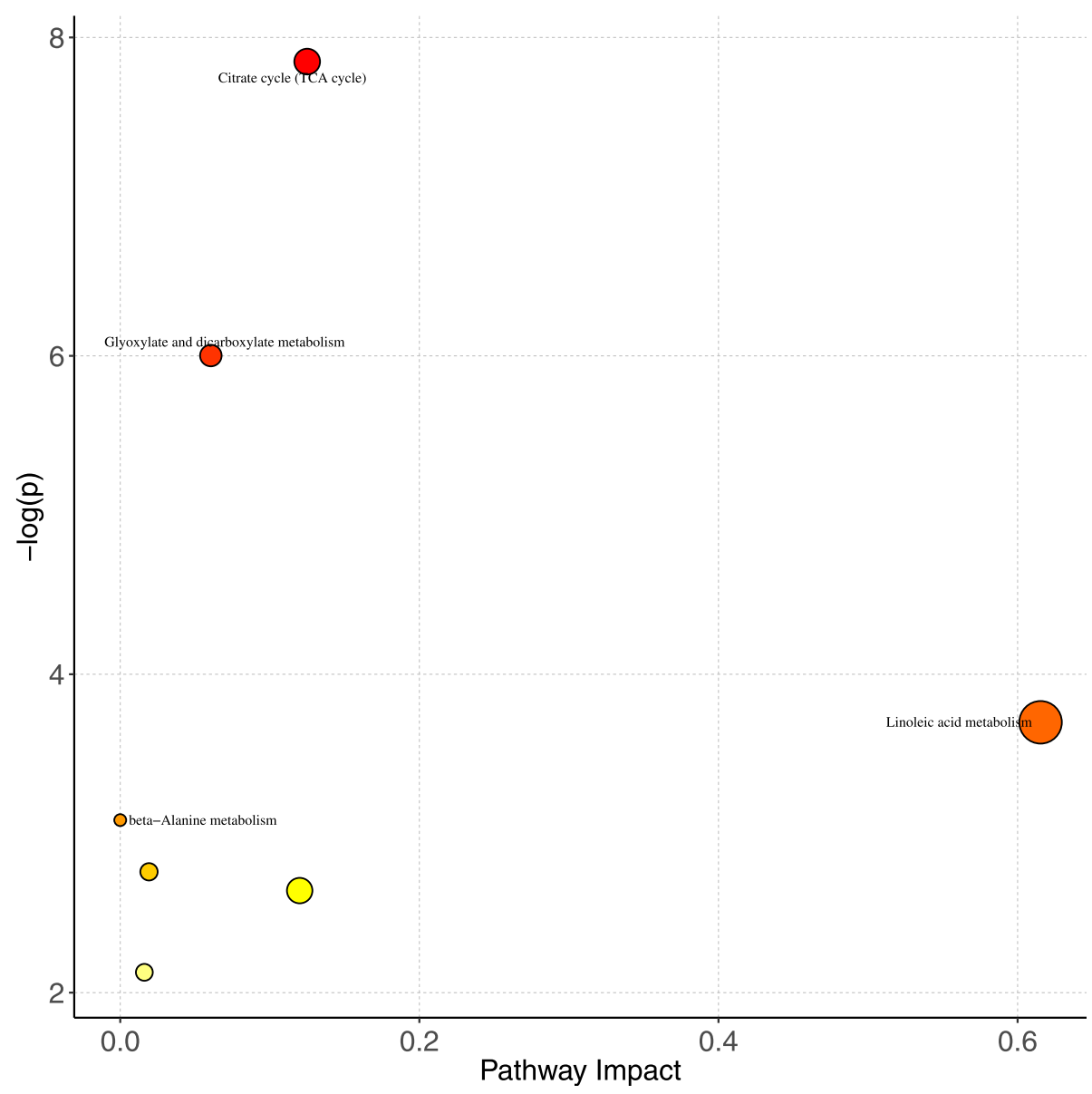

Fig. 3 Pathway analysis of the differential metabolites between the pCR and non-pCR groups. The altered pathway of ESCC

chemoradiosensitivity-associated metabolites. The color and size of each circle is based on P value and pathway impact value respectively

citrate cycle (TCA cycle), glyoxylate and dicarboxylate metabolism were significantly altered between $\mathrm{pCR}$ and non-pCR (Fig. 3 and Table 2).

In the previous study, altered metabolic profile was found between the pCR and non-pCR ESCC patients in the serum level detected by gas chromatography mass spectrometry (GC/MS) analysis and liquid chromatography mass spectrometry (LC/MS) analysis [21]. But only three differential metabolites (arabitol, uracil and 3-aminoglutaric acid) were detected by $\mathrm{GC} /$ MS analysis. While in the present research, five differential metabolites, including citric acid and isocitric acid were identified as plasma biomarker candidates for chemoradiosensitivity applied by GC-TOF/MS analysis.

Table 2 Pathway analysis of metabolite changes

\begin{tabular}{|c|c|c|c|c|c|}
\hline & Total In Pathway & Hits & $\boldsymbol{p}$ Value & Impact & $\begin{array}{l}\text { Enriched } \\
\text { Compounds }\end{array}$ \\
\hline Citrate cycle (TCA cycle) & 20 & 2 & 0.00038978 & 0.125 & $\begin{array}{l}\text { Citric acid } \\
\text { Isocitric acid }\end{array}$ \\
\hline Glyoxylate and dicarboxylate metabolism & 50 & 2 & 0.0024713 & 0.0606 & $\begin{array}{l}\text { Citric acid } \\
\text { Isocitric acid }\end{array}$ \\
\hline Linoleic acid metabolism & 15 & 1 & 0.024711 & 0.61538 & Linoleic acid \\
\hline beta-Alanine metabolism & 28 & 1 & 0.045753 & 0 & L-Histidine \\
\hline Nitrogen metabolism & 39 & 1 & 0.063291 & 0.01923 & L-Histidine \\
\hline Histidine metabolism & 44 & 1 & 0.071183 & 0.12 & L-Histidine \\
\hline Aminoacyl-tRNA biosynthesis & 75 & 1 & 0.119 & 0.01613 & L-Histidine \\
\hline
\end{tabular}


There is no evidence that plasma or serum was superior to be applied in clinical metabolomic study. However, there are indeed differential distributions of specific metabolites between plasma and serum. For instance, erythritol, glycerophosphocholines, glutamine, creatinine, and hexadecanoic acid in plasma, but not in serum, were shown to link with life expectancy for small-cell lung cancer patients [31]. In addition, another reason including chemical diversity and pretreatment of sample may also have impact on the disparate results. For example, citrate and citrate cycle metabolites were found in relative lower value in serum, partially because citrate in serum forms divalent cation complexes, which will precipitate by methanol extraction method [32]. Our study provided another dimension in investigation of the metabolites associated with nCRT respondence in the plasma level.

In the present study, L-histidine was found downregulated in the pCR patients. Previous study showed that increasing serum level of histidine was observed in breast cancer patient and strongly related with disease relapse [33]. Moreover, histidine degradation was found to be able to enhance the sensitivity to cancer therapy [34]. Meanwhile, recent study reported that high expression of FAM83A predicted a poor prognosis in lung adenocarcinoma patients, and histidine metabolism pathway was found significantly activated in FAM83A high expressed lung adenocarcinoma sample [35]. It is considered that the marked gain of histidine may enhance the total antioxidant and metal-binding capacity of the proteome of the cancer cell and thus potentially serve as a nonspecific compensatory mechanism to relieve consequences of the cancer-related aggravation of oxidative stress [36]. Furthermore, according to the pathway analysis, the tricarboxylic acid (TCA) cycle and the glyoxylate and dicarboxylate metabolism were found significantly related to ESCC chemoradiosensitivity. The TCA cycle is a key process for energy generation, which consumes oxygen and generates high amounts of ATP through oxidative phosphorylation. While for cancer cells, glycolysis is the primary pathway for energy production even if oxygen is available. Glycolytic pyruvate prefers to be converted into lactate, rather than enter into the mitochondrial TCA cycle. This phenomenon is called aerobic glycolysis or the Warburg effect. This kind of aberration has been proved to afford biosynthetic precursors for rapid macromolecule synthesis, and to keep cellular redox homeostasis for better survival [37, 38]. Mounting studies have demonstrated that there is a relationship between aerobic glycolysis and the occurrence of tumor drug resistance. And several key proteins in the glycolytic pathway have been discovered as promising targets for overcoming chemoresistance [39-41]. It was reported that suppressing glycolytic enzymes by inhibiting RAC1 showed reduced cisplatin resistance in esophageal squamous cell carcinoma [42]. Meanwhile, pretreatment whole-body total lesion glycolysis was uncovered as an independent predictor of outcomes in patients with esophageal cancer treated with definitive chemoradiotherapy [43]. In addition, altered glyoxylate and dicarboxylate metabolic pathway have been reported in various cancer. Evidence showed that glyoxylate and dicarboxylate metabolism was associated with the loss of tumor cell differentiation in lung adenocarcinomas [44]. Current study suggested that glyoxylate and dicarboxylate metabolism combined with other pathways could distinguish tumor from normal tissues in colorectal cancer [45]. In gastric cancer, glyoxylate and dicarboxylate metabolism were observed in the chromosomal instability type alone [46]. While previous study demonstrated that chromosomal instability is a favorable predictor of response to cisplatin-based neoadjuvant chemotherapy in patients [47]. In combination with the findings in this study, citric acid and isocitric acid, as the intermediates of the TCA cycle and the glyoxylate and dicarboxylate metabolic pathway, were up-regulated in the pCR patients. It is suggested that the energy metabolism was involved in the regulatory mechanism of sensitivity to neoadjuvant therapy for esophageal cancer.

There also exist some limitations in this study. The primary limitation is that this is a single-center study with relatively small number of subjects, making the data less conclusive. In addition, due to the restriction of the conditions, we only applied one metabolomics method to identify the potential changed metabolites. Furthermore, the predictive precision of those differential metabolites needed external validation. Larger samples and a combination of research methods may help us better understand the mechanism of ESCC chemoradiotherapy resistance.

\section{Conclusion}

Overall, we found that significant alteration of metabolites between the responders and non-responders ESCC patients who received neoadjuvant chemoradiotherapy. The pCR group exhibited higher level of isocitric acid, linoleic acid, citric acid, and lower level of L-histidine, 3'4 dihydroxyhydrocinnamic acid than the non-pCR group. Changes in plasma metabolic signature may reflect reprogramming of the aforementioned metabolic pathways. Further study is needed to validate these findings using larger samples and to explore the underlying mechanism of ESCC chemoradiotherapy resistance. 


\section{Supplementary information}

Supplementary information accompanies this paper at https://doi.org/10. 1186/s12885-020-07336-9.

Additional file 1.

Additional file 2: Supplementary Figure 1. Metabolic network of the changed metabolites and altered metabolic pathways in KEGG general metabolic pathway map. Red dots represent the increased metabolites in pCR group; Blue dots represent the specifically decreased metabolites in pCR group.

\section{Abbreviations}

AUC: Area under the curve; EC: Esophageal cancer; ESCC: Esophageal squamous cell carcinoma; GC-TOF/MS: Gas chromatography time-of-flight mass spectrometry; GC/MS: Gas chromatography mass spectrometry; LC/ MS: Liquid chromatography mass spectrometry; nCRT: Neoadjuvant chemoradiotherapy; pCR: Pathological complete response; ROC: Receiver operating characteristic; TCA: Tricarboxylic acid; TNM: Tumor-node-metastasis

\section{Acknowledgments}

We thank all of patients for their participation in the study and their doctors for their willingness to refer patients.

\section{Authors' contributions}

$F Z, J H Z$ and $Y Z$ conceived and designed the study. YZ, JW, ND, PH and JL collected the clinical data. YZ, JW and ND performed the experiments. WY and $\mathrm{JMZ}$ analyzed the data. $\mathrm{JHZ}$ and $\mathrm{YZ}$ wrote the manuscript. All authors have read and approved the manuscript.

\section{Funding}

This study was supported by National Natural Science Foundation of China (U1504814), Major projects of science and Technology Department in Henan Province (16110311200, 161100311300) and Anyang Science Foundation of Henan province (2016). The funders had no role in study design, data collection and analysis, decision to publish, or preparation of the manuscript.

\section{Availability of data and materials}

All the necessary materials can be found in the text or supplementary materials. Due to the privacy policy, the confidential data materials could only be obtained with the permission of the corresponding authors.

\section{Ethics approval and consent to participate}

This study was approved by the Ethics Committee of Anyang Cancer Hospital, Chinese Academy of Medical Sciences, and all human subjects signed informed consent before participating in this study.

\section{Consent for publication}

Not applicable.

\section{Competing interests}

The authors declare that there are no conflicts of interest.

Received: 13 April 2020 Accepted: 24 August 2020

Published online: 02 September 2020

\section{References}

1. Bray F, Ferlay J, Soerjomataram I, Siegel RL, Torre LA, Jemal A. Global cancer statistics 2018: GLOBOCAN estimates of incidence and mortality worldwide for 36 cancers in 185 countries. CA Cancer J Clin. 2018:68:394-424.

2. Shen FF, Pan Y, Yang HJ, Li JK, Zhao F, Su JF, et al. Decreased expression of SPINT1-AS1 and SPINT1 mRNA might be independent unfavorable prognostic indicators in esophageal squamous cell carcinoma. OncoTargets Ther. 2019;12:4755-63.

3. Chen MF, Chen PT, Lu MS, Lee CP, Chen WC. Survival benefit of surgery to patients with esophageal squamous cell carcinoma. Sci Rep. 2017;7:46139.

4. D'Amico TA. Outcomes after surgery for esophageal cancer. Gastrointestinal Cancer Res. 2007;1:188-96.

5. Meves V, Behrens A, Pohl J. Diagnostics and early diagnosis of esophageal Cancer. Viszeralmedizin. 2015;31:315-8.
6. Shapiro J, van Lanschot JJB, Hulshof $M$, van Hagen $P$, van Berge Henegouwen Ml, Wijnhoven BPL, et al. Neoadjuvant chemoradiotherapy plus surgery versus surgery alone for oesophageal or junctional cancer (CROSS): long-term results of a randomised controlled trial. Lancet Oncol. 2015;16:1090-8

7. Yang H, Liu H, Chen Y, Zhu C, Fang W, Yu Z, et al. Neoadjuvant Chemoradiotherapy followed by surgery versus surgery alone for locally advanced squamous cell carcinoma of the esophagus (NEOCRTEC5010): a phase III multicenter, randomized, open-label clinical trial. J Clin Oncol. 2018;36:2796-803.

8. van Hagen P, Hulshof MC, van Lanschot JJ, Steyerberg EW, van Berge Henegouwen MI, Wijnhoven BP, et al. Preoperative chemoradiotherapy for esophageal or junctional cancer. N Engl J Med. 2012;366:2074-84.

9. Sato Y, Motoyama S, Saito H, Minamiya Y. Novel candidate biomarkers of Chemoradiosensitivity in esophageal squamous cell carcinoma: a systematic review. Eur Surg Res. 2016;56:141-53.

10. Borggreve AS, Mook S, Verheij M, Mul VEM, Bergman JJ, Bartels-Rutten A, et al. Preoperative image-guided identification of response to neoadjuvant chemoradiotherapy in esophageal cancer (PRIDE): a multicenter observational study. BMC Cancer. 2018;18:1006.

11. Hsu PK, Chien LI, Huang CS, Hsieh CC, Wu YC, Hsu WH, et al. Comparison of survival among neoadjuvant chemoradiation responders, non-responders and patients receiving primary resection for locally advanced oesophageal squamous cell carcinoma: does neoadjuvant chemoradiation benefit all? Interact Cardiovasc Thorac Surg. 2013;17:460-6.

12. Mamas M, Dunn WB, Neyses L, Goodacre R. The role of metabolites and metabolomics in clinically applicable biomarkers of disease. Arch Toxicol. 2011;85:5-17.

13. Spratlin JL, Serkova NJ, Eckhardt SG. Clinical applications of metabolomics in oncology: a review. Clin Cancer Res. 2009;15:431-40.

14. Puchades-Carrasco L, Pineda-Lucena A. Metabolomics applications in precision medicine: an oncological perspective. Curr Top Med Chem. 2017; 17:2740-51

15. Qiu Y, Cai G, Zhou B, Li D, Zhao A, Xie G, et al. A distinct metabolic signature of human colorectal cancer with prognostic potential. Clin Cancer Res. 2014;20:2136-46.

16. Alberice JV, Amaral AF, Armitage EG, Lorente JA, Algaba F, Carrilho E, et al. Searching for urine biomarkers of bladder cancer recurrence using a liquid chromatography-mass spectrometry and capillary electrophoresis-mass spectrometry metabolomics approach. J Chromatogr A. 2013;1318:163-70.

17. Mock A, Zschabitz S, Kirsten R, Scheffler M, Wolf B, Herold-Mende C, et al. Serum very long-chain fatty acid-containing lipids predict response to immune checkpoint inhibitors in urological cancers. Cancer Immunol Immunother. 2019;68:2005-14.

18. Davis VW, Schiller DE, Eurich D, Sawyer MB. Urinary metabolomic signature of esophageal cancer and Barrett's esophagus. World J Surg Oncol. 2012:10:271

19. Tokunaga M, Kami K, Ozawa S, Oguma J, Kazuno A, Miyachi H, et al. Metabolome analysis of esophageal cancer tissues using capillary electrophoresis-time-of-flight mass spectrometry. Int J Oncol. 2018;52: 1947-58.

20. Buas MF, Gu H, Djukovic D, Zhu J, Onstad L, Reid BJ, et al. Candidate serum metabolite biomarkers for differentiating gastroesophageal reflux disease, Barrett's esophagus, and high-grade dysplasia/esophageal adenocarcinoma. Metabolomics. 2017;13(3). https://doi.org/10.1007/s11306-016-1154-y.

21. Fujigaki S, Nishiumi S, Kobayashi T, Suzuki M, lemoto T, Kojima T, et al. Identification of serum biomarkers of chemoradiosensitivity in esophageal cancer via the targeted metabolomics approach. Biomark Med. 2018;12:827-40.

22. Rice TW, Ishwaran H, Ferguson MK, Blackstone EH, Goldstraw P. Cancer of the esophagus and Esophagogastric junction: an eighth edition staging primer. J Thoracic Oncol. 2017;12:36-42.

23. Chiu CH, Zhang P, Chang AC, Derstine BA, Ross BE, Enchakalody B, et al. Morphomic factors associated with complete response to Neoadjuvant therapy in esophageal carcinoma. Ann Thorac Surg. 2020;109:241-8.

24. Wang Y, Zhao H, Liu Y, Guo W, Bao Y, Zhang M, et al. GC-MS-based metabolomics to reveal the protective effect of gross Saponins of Tribulus terrestris fruit against ischemic stroke in rat. Molecules. 2019;24. https://doi. org/10.3390/molecules24040793.

25. Xi Y, Yuefen P, Wei W, Quan Q, Jing Z, Jiamin X, et al. Analysis of prognosis, genome, microbiome, and microbial metabolome in different sites of colorectal cancer. J Transl Med. 2019;17:353. 
26. Yu Y, Gao Z, Lou J, Mao Z, Li K, Chu C, et al. Identification of serum-based metabolic feature and characteristic metabolites in Paraquat intoxicated mouse models. Front Physiol. 2020;11:65.

27. Ping F, Guo Y, Cao Y, Shang J, Yao S, Zhang J, et al. Metabolomics analysis of the renal cortex in rats with acute kidney injury induced by sepsis. Front Mol Biosci. 2019;6:152.

28. Francis AM, Sepesi B, Correa AM, Blum MA, Erasmus JJ, Lee JH, et al. The influence of histopathologic tumor viability on long-term survival and recurrence rates following neoadjuvant therapy for esophageal adenocarcinoma. Ann Surg. 2013:258:500-7.

29. Jaeger C, Meret M, Schmitt CA, Lisec J. Compound annotation in liquid chromatography/high-resolution mass spectrometry based metabolomics: robust adduct ion determination as a prerequisite to structure prediction in electrospray ionization mass spectra. Rapid Commun Mass Spectrom. 2017; 31:1261-6.

30. Yin S, Guo P, Hai D, Xu L, Shu J, Zhang W, et al. Optimization of GC/TOF MS analysis conditions for assessing host-gut microbiota metabolic interactions: Chinese rhubarb alters fecal aromatic amino acids and phenol metabolism. Anal Chim Acta. 2017:995:21-33.

31. Wedge DC, Allwood JW, Dunn W, Vaughan AA, Simpson K, Brown M, et al. Is serum or plasma more appropriate for intersubject comparisons in metabolomic studies? An assessment in patients with small-cell lung cancer. Anal Chem. 2011;83:6689-97.

32. Dettmer K, Almstetter MF, Appel IJ, Nurnberger N, Schlamberger G, Gronwald W, et al. Comparison of serum versus plasma collection in gas chromatography--mass spectrometry-based metabolomics. Electrophoresis. 2010;31:2365-73.

33. Tenori L, Oakman C, Morris PG, Gralka E, Turner N, Cappadona S, et al. Serum metabolomic profiles evaluated after surgery may identify patients with oestrogen receptor negative early breast cancer at increased risk of disease recurrence. Results from a retrospective study. Mol Oncol. 2015;9: 128-39.

34. Kanarek N, Keys HR, Cantor JR, Lewis CA, Chan SH, Kunchok T, et al. Histidine catabolism is a major determinant of methotrexate sensitivity. Nature. 2018;559:632-6.

35. Yu J, Hou M, Pei T. FAM83A is a prognosis signature and potential oncogene of lung adenocarcinoma. DNA Cell Biol. 2020;39:890-9.

36. Tsuber V, Kadamov Y, Brautigam L, Berglund UW, Helleday T. Mutations in Cancer cause gain of cysteine, Histidine, and tryptophan at the expense of a net loss of arginine on the proteome level. Biomolecules. 2017;7. https:// doi.org/10.3390/biom7030049.

37. Sun L, Suo C, Li ST, Zhang H, Gao P. Metabolic reprogramming for cancer cells and their microenvironment: beyond the Warburg effect. Biochim Biophys Acta Rev Cancer. 1870;2018:51-66.

38. Pavlova NN, Thompson CB. The emerging hallmarks of Cancer metabolism. Cell Metab. 2016;23:27-47.

39. Sfakianaki M, Papadaki C, Tzardi M, Trypaki M, Manolakou S, Messaritakis I, et al. PKM2 expression as biomarker for resistance to oxaliplatin-based chemotherapy in colorectal cancer. Ann Oncol. 2019; 30 Suppl 4:iv21-iv2.

40. Avolio R, Matassa DS, Criscuolo D, Landriscina M, Esposito F. Modulation of mitochondrial metabolic reprogramming and oxidative stress to overcome Chemoresistance in Cancer. Biomolecules. 2020;10. https://doi.org/10.3390/ biom10010135.

41. Abdel-Wahab AF, Mahmoud W, Al-Harizy RM. Targeting glucose metabolism to suppress cancer progression: prospective of anti-glycolytic cancer therapy. Pharmacol Res. 2019;150:104511.

42. Zeng RJ, Zheng CW, Gu JE, Zhang HX, Xie L, Xu LY, et al. RAC1 inhibition reverses cisplatin resistance in esophageal squamous cell carcinoma and induces downregulation of glycolytic enzymes. Mol Oncol. 2019;13:2010-30

43. Takahashi N, Umezawa R, Takanami K, Yamamoto T, Ishikawa Y, Kozumi M, et al. Whole-body total lesion glycolysis is an independent predictor in patients with esophageal cancer treated with definitive chemoradiotherapy. Radiother Oncol. 2018;129:161-5.

44. Creighton C, Hanash S, Beer D. Gene expression patterns define pathways correlated with loss of differentiation in lung adenocarcinomas. FEBS Lett. 2003:540:167-70

45. Arima K, Lau MC, Zhao M, Haruki K, Kosumi K, Mima K, et al. Metabolic profiling of formalin-fixed paraffin-embedded tissues discriminates Normal Colon from colorectal Cancer. Mol Cancer Res. 2020;18:883-90.

46. Tsai CK, Yeh TS, Wu RC, Lai YC, Chiang MH, Lu KY, et al. Metabolomic alterations and chromosomal instability status in gastric cancer. World J Gastroenterol. 2018;24:3760-9.

47. Ott K, Vogelsang H, Mueller J, Becker K, Muller M, Fink U, et al. Chromosomal instability rather than p53 mutation is associated with response to neoadjuvant cisplatin-based chemotherapy in gastric carcinoma. Clin Cancer Res. 2003;9:2307-15.

\section{Publisher's Note}

Springer Nature remains neutral with regard to jurisdictional claims in published maps and institutional affiliations.
Ready to submit your research? Choose BMC and benefit from:

- fast, convenient online submission

- thorough peer review by experienced researchers in your field

- rapid publication on acceptance

- support for research data, including large and complex data types

- gold Open Access which fosters wider collaboration and increased citations

- maximum visibility for your research: over $100 \mathrm{M}$ website views per year

At BMC, research is always in progress.

Learn more biomedcentral.com/submissions 